\title{
A Comparative Study on the Effect of Eccentric Viewing Training Using PC and VR Contents
}

\author{
Dokyeong Lee ${ }^{\mathrm{a},{ }^{*}}$, HwaMin Lee ${ }^{\mathrm{b}}$ \\ ${ }^{a}$ Department ICT Convergence Rehabilitation Engineering, Soonchunhyang University, Asan, South Korea \\ ${ }^{b}$ Department Computer Software \& Engineering, Soonchunhyang University, Asan, South Korea \\ Corresponding author: *leehm@sch.ac.kr
}

\begin{abstract}
The purpose of this study is to develop PC Eccentric Viewing Training (EVT) and VR EVT Content, to conduct experiments to verify the validity of contents first through non-disabled people, and to compare and analyze VR and PC contents. Both PC and VR contents were produced with UNITY. This content model assumes that reducing inaccurate saccades by eliminating eye movement helps improve reading accuracy. In addition, the two contents are implemented in the same way as VR and PC versions. The content consists of two steps, both PC and VR. The purpose of the content is to improve reading accuracy by improving the fixation stability of Preferred Retinal Locus (PRL) and reducing inaccurate Saccades. The experiment consisted of 12 persons (within maximum visual acuity less than 0.3), and they were assigned to the PC Content group and VR Content group. The experiment was conducted a total of 5 times, except for two weeks, which is the time to adapt PRL. The experimental results showed that the reading accuracy of the VR content group was higher. In addition, When comparing VR contents with PC contents, the group that conducted the training through PC contents showed a decrease in concentration as it progressed to 1-3 steps, and the score distribution also fell overall. In conclusion, the study compared VR and PC contents, and the effectiveness of contents was verified through experiments.
\end{abstract}

Keywords-Eccentric Viewing Training; low vision people with scotoma; preferred retinal locus; Macular degeneration; visual rehabilitation content.

\section{INTRODUCTION}

The visual system can be divided into a central vision and a peripheral vision. Reading detailed and accurate information is the central vision's role, and the macula plays a large part in the role [1], [2]. The area within 5 (degree) where the optic nerve is concentrated plays a function of distinguishing face recognition, distance perception, color, and shape [3]-[5]. The peripheral vision's role serves to detect a wide background or moving objects [2], [3]. If the function of the central vision is impaired due to macular degeneration, the peripheral vision replaces the function of the central vision [2], [6], [7]. They are included in low vision, and visual acuity reduction due to macular disorders, central scotoma, and distorted objects [3], [8]. Treatment and rehabilitation are required because low vision patients with these symptoms may be exposed to accidents such as falls and fractures due to the unsystematic visual range [9].
Recently, the possibility of preventing worsening of symptoms and maintaining residual vision has increased with various treatments due to the development of medical technologies such as anti-vascular growth factor antibody injection and photodynamic therapy. However, it is currently impossible to cure, and mostly continuous treatment and rehabilitation are required [10]. In the case of visual rehabilitation to preserve residual vision, visual rehabilitation's timing has a decisive effect on prognosis. In addition, studies on the effects of visual rehabilitation have been reported continuously [2], [3], [11]-[14], but since the effects are semi-permanent, constant and continuous training is necessary. In Korea, the basis for rehabilitation for lowvision people is insufficient, so low-vision people are experiencing difficulties in rehabilitation [2]. Currently, the visual field disorder in Korea is only $1.4 \%$ based on the registered persons with disabilities, but macular degeneration is likely to increase with the aging of the eye [13], [15], [16]. 
People with macular disorders have difficulties in a variety of daily lives [8], [17]-[20]. As a way to overcome this obstacle, a combination of visual rehabilitation and the use of optical aids in adaptive strategies [21], [22] such as peripheral vision training and eccentric fixation stability is required [23]. The most effective rehabilitation method is to maximize the rehabilitation effect through optical aid after conducting Eccentric Viewing Training (EVT) [2].

EVT is ongoing training for patients with visual field defects and training to better view images formed through residual vision [23]. In patients with central visual field loss, the preferred retinal locus (PRL) is naturally selected to perform EVT [1], [23]. PRL has been known to exist for many years [2], [21], [24], and the recent research trend is to identify PRLs for EVT or use Training retinal locus (TRL) [25]. Through rehabilitation through EVT, it is not possible to reconcile peripheral vision as much as the vision of the central vision. However, it is possible to reduce the degree of discomfort in life to a certain extent by continuously training the subject in detail with a peripheral vision [11], [12]

Although the existing EVT rehabilitation method was conducted through a visit to a rehabilitation center, recently, research on home vision therapy, which enables rehabilitation at home due to software and device diversification, is also active [13], [14]. In particular, as VR technology has rapidly developed in recent years, it has started to be grafted into various education and training fields, and research on various rehabilitation methods based on VR is also ongoing [26]-[29].

Many previous papers have said that the attractive components of VR can contribute to successful results [30][32]. In addition, when focusing on the theoretical background that virtual reality enables visual-electric static interaction by rich visual stimuli, virtual reality is likely to provide an optimized environment for better performance in VR rehabilitation [28], [31], [32]. In the future, the field of VR-based rehabilitation content is likely to expand further, and the research is expected to become more active.

In this study, the primary goal is to implement content for low vision people with scotoma due to macular degeneration. In addition, it aims to conduct experiments for primary effectiveness verification through non-disabled people. Eccentric Viewing Training is a rehabilitation training that adapts to view the target with peripheral vision. It also combines training to improve eccentric fixation stability using PRL and Oculomotor training [21], [33], [34].

Various studies on Eccentric Viewing Training and PRL have been conducted. Persons who have lost the Central Vision Loss (CVL) naturally develop the cortical reorganization of visual processing in the calcarine sulcus to improve visual function [7]. This is the reason that most of the CVLs choose PRLs for viewing peripheral vision [1]. It is possible that there may be more than one PRL, and it may be variable before receiving a fixed PRL [35]. Even with patients with sudden vision limitations or patients with PRL, many patients are unaware that their vision is not fixed to the target [36]. If the target is not accurately matched with the PRL, the time to recognize and inaccurate Saccades increases [19]. Currently, studies on adaptation of the peripheral vision to fix these problems and fixation of the PRL are actively underway.
RSVP proceeds by following the reading using the possible PRL function without using eye movement. While the gaze is fixed at the center of the screen, one word is displayed on the screen monitor. Many previous studies have used the RSVP training method for rehabilitation [3], [37], [38]. Deyue $\mathrm{Yu}$ [38] conducted a study to improve the reading speed of lost people with scotoma. Twenty-seven non-disabled people were divided into the lexical-decision training group, trigram letter-recognition training group, and RSVP training group. They trained $10^{\circ}$ in the lower visual field, and it was reported that RSVP (Rapid Serial Visual Presentation) training was effective in improving reading speed. Seung-Pyo Hong [3] experimented by implementing Korean-based eccentric viewing software that could be applied to patients with scotoma. This program consisted of 5 stages, three stages of word reading training and twosentence reading training stages. The experiment results with two non-disabled male college students reported that Korean-based EVT's effectiveness could be confirmed.

\section{MATERIAL AND METHOD}

This content was produced in two versions to test the hypothesis that PRL fixation stability's improvement affects the target's attention and reading. Since the PC version cannot set constraints, it is affected by the surrounding environment and requires the help of experts or assistants to maximize the rehabilitation process, results, and effects. However, in the case of rehabilitation through VR content, limitations of visibility and constraints required for performing various rehabilitation can be added through HMD. This way, trainees can perform rehabilitation with minimal assistance. FOVE HMD was used as the hardware for the VR content [39]. Fig. 1 shows the flow chart of contents progress.

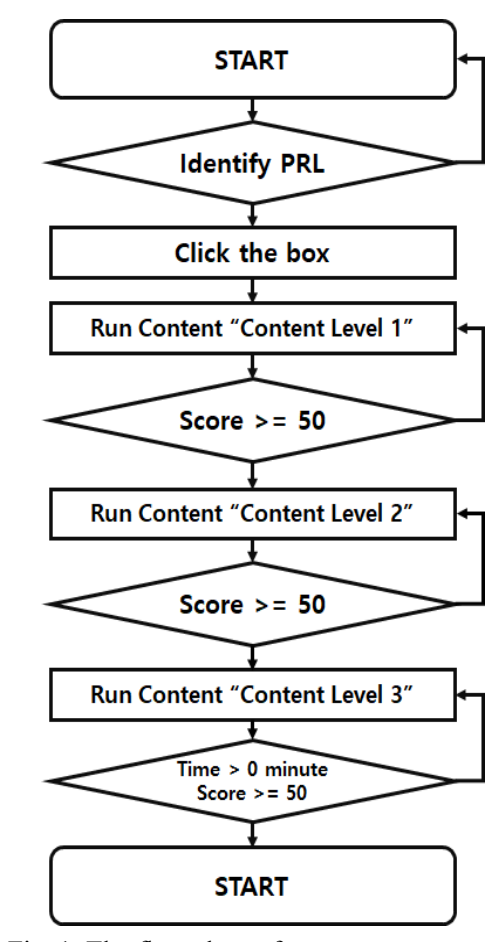

Fig. 1 The flow chart of contents progress

Both contents are conducted in RSVP format and consist of simple arrow shapes and numbers. The VR and PC 
contents were trained on the right and left eyes alternately, and the view field on the non-training side was covered. Content is commonly measured time limits, scores, etc., and the content ends when the time limit expires or when the score goes down to 0 points.

The composition of the rehabilitation content was implemented using the method devised from the method of rehabilitation used in the previous study experiments [14], [40]. In the first stage game, the target's size was adjusted to about 40 points, the second stage was divided into about 30 points, and the third stage was divided into size stages of about 20 points. If the target displayed on the screen is corrected, the score of 2 points increases, and if the target is wrong or cannot be matched, it is calculated that the score of 1 point decreases. Below is a flowchart of each content of VR and PC.

When the PC content starts, the boxes (Left in Fig. 2) are presentation and disappear in order. The process is continued until the user selects the most visible field of view. When the content Level 1 starts, the user selects the right direction through the keyboard's arrows. When the score reaches 50 points or more, or over 60 seconds, the content Level 2 starts with the target size reduced. Level 2 and 3 proceed in the same way. Content 1, 2 and 3 have only different target sizes, and there is no change in the target presentation time and content running time.

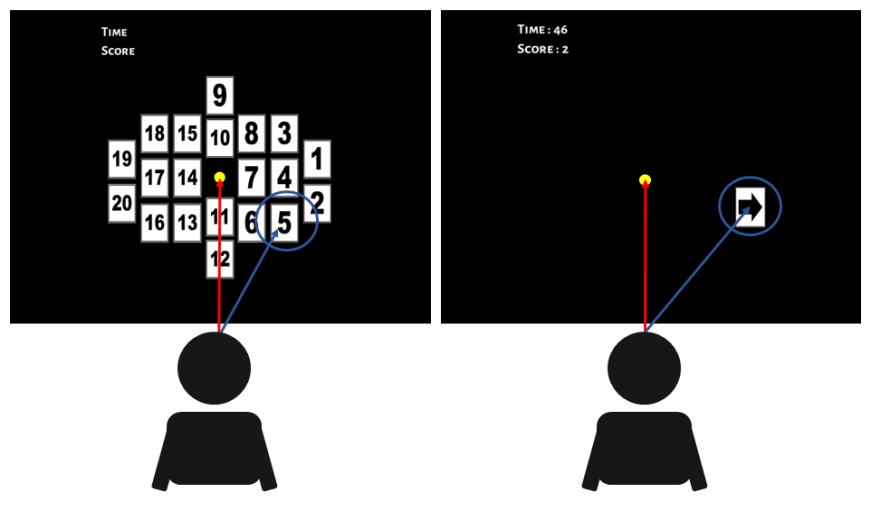

Fig. 2 Identify PRL(Left), PC Contents run(Right)

\section{A. PC Contents}

In the case of PC contents, the user checks the center point before starting the contents and then induces the user to look at the point. After that, when the content starts, the boxes are displayed in order and disappear, and the user can select the PRL by tapping the most visible box through the surrounding field of view. Thereafter, an arrow is randomly generated in the area of the box selected with the PRL, and the score increases when the correct arrow direction is input through the keyboard, and the score decreases when the arrow in the other direction is input. The speed and size at which the object of the arrow appears can be increased or decreased through user or assistant manipulation.

Fig. 2 is a picture of the actual content. The left in Fig. 2 is the process of selecting the PRL. If the area most visible when the trainee is looking at the center dot point is a box of 5, click that area. The right side of Fig. 2 shows the progress of the content. If number 5 is PRL, the content starts in the area of the number 5 .

\section{B. VR Contents}

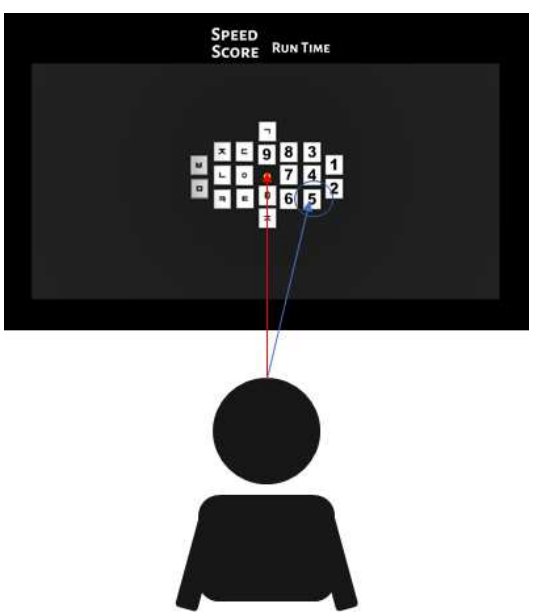

Fig. 3 Identify PRL in content

As shown in Fig. 3, the VR contents also check the PRL in the same way, and then the content starts. When run VR content, Trainees should look at the center point, the same as $\mathrm{PC}$ content, and choose the number that looks best.
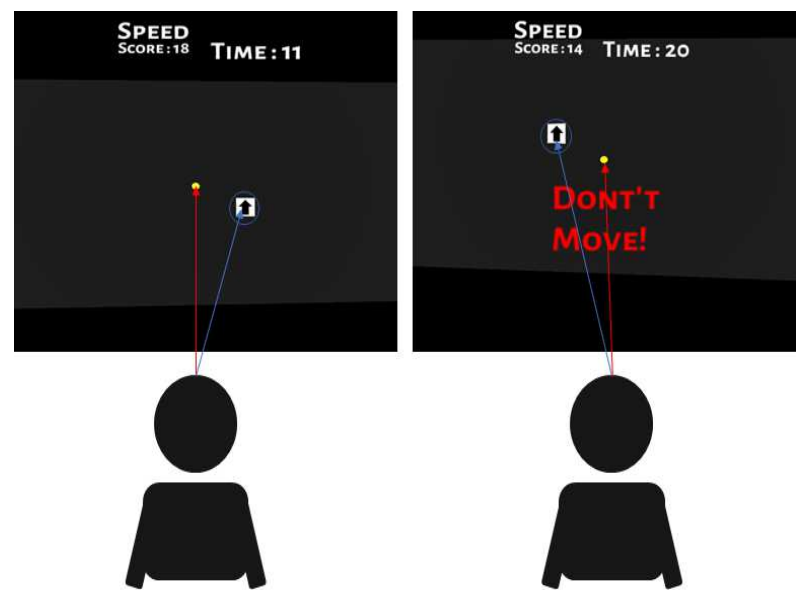

Fig. 4 VR content run. Not moving of head (Left) when the trainee's head move(Right)

When starting the content, a target point that the trainee should look at is presented. The trainer must look at the point and use the peripheral vision to look at the target (Fig. 4). If the trainee's eyes do not accurately look at the target point, the content is stopped, and the training is set to proceed again only when the trainee properly looks at the target point. The VR device used FOVE, and the trainee's eye movement was measured through the EYE Tracking function built into the device. The result of the measurement was expressed as the time of looking at the target accurately and the distribution of the eyes.

As shown in Fig. 4, If the trainee's head moves during training, a warning message (right in Fig. 4) is displayed so that the object can be seen only by a peripheral vision where PRL without eye movement and moving the head. The warning message was displayed at the center of the user's screen when the head moved out from 20 to 340 degrees in the $\mathrm{X}$-axis, from 20 to 360 degrees on the Y-axis, and 10 to 35 degrees Z-axis.

This study devised Chen [39] research before clinical application to macular degeneration patients and conducted 
experiments with low vision patients. This experiment is to report how the improvement of fixation stability of PRL affects the accuracy of viewing the target through nondisabled people with low vision. The experimenter consisted of 12 non-disabled students (within maximum visual acuity of 0.3). Table I shows information about the experiment participants of VR contents and PC contents. The first chart is the VR and the second chart is the PC (table I).

\section{RESULT AND DISCUSSION}

Before the experiment, all participants were given informed consent. In order to proceed with the investigation, experimenters made a point to see in the center of PC and VR contents. The content progress was 3 minutes per section and 1 time a day. Between sections, sufficient eye rest was provided, and if the participant complained of discomfort in the middle of the content, the experiment was immediately stopped. The results of the experiment are presented in tables and graphs.

TABLE I

PROFILE OF SUBJECTS OF VR CONTENTS AND PC CONTENTS

\begin{tabular}{ccc}
\hline \multirow{2}{*}{ ID } & \multicolumn{2}{c}{ Healthy Subject } \\
\cline { 2 - 3 } & Gender & Age \\
\hline H1 & M & 29 \\
H2 & M & 26 \\
H3 & M & 26 \\
H4 & F & 28 \\
H5 & F & 23 \\
H6 & F & 26 \\
H7 & M & 32 \\
H8 & M & 29 \\
H9 & M & 27 \\
H10 & F & 24 \\
H11 & F & 24 \\
H12 & F & 25 \\
\hline
\end{tabular}

In the process of determining the fixed PRL of nondisabled test participants, the center point was instructed to select the most visible area. The period for determining the fixed PRL was two weeks, and an attempt was made to confirm the PRL once a day. During the two-week PRL selection period, two of the study participants were similarly employed in more than two PRL regions. It was found that macular degeneration patients may form more than one PRL when forming PRL through sudden vision loss. Among the selected PRL areas for two weeks, it was assumed that the area with a large number of overlapping selections was a PRL, but it was also changed when requested by the experimenter. In the experiment's data and period, the period during which the participant finds a fixed PRL is excluded from the data. However, when the variation of PRL BOX selection decreases in the process of determining the fixed PRL before the experiment participants proceed with the experiment, the area was predicted as the region of the experiment participants.

\section{A. Result of VR Contents}

From Table II to Table VII is the result score of participants of VR content. The score was averaged after measuring the right and left eye scores separately.
TABLE II

THE SCORE OF H1

\begin{tabular}{cccccc}
\hline Content & \multicolumn{5}{c}{ Training Date and Score } \\
\cline { 2 - 6 } Levels & Day 1 & Day 2 & Day 3 & Day 4 & Day 5 \\
\hline Level 1 & 37 & 34 & 40 & 38 & 44 \\
Level 2 & 32 & 33 & 30 & 38 & 37 \\
Level 3 & 20 & 32 & 28 & 35 & 37 \\
\hline
\end{tabular}

TABLE III

THE SCORE OF H2

\begin{tabular}{cccccc}
\hline Content & \multicolumn{5}{c}{ Training Date and Score } \\
\cline { 2 - 6 } Levels & Day 1 & Day 2 & Day 3 & Day 4 & Day 5 \\
\hline Level 1 & 35 & 34 & 31 & 35 & 34 \\
Level 2 & 35 & 32 & 34 & 38 & 39 \\
Level 3 & 18 & 20 & 17 & 15 & 16 \\
\hline
\end{tabular}

TABLE IV

THE SCORE OF H3

\begin{tabular}{cccccc}
\hline Content & \multicolumn{5}{c}{ Training Date and Score } \\
\cline { 2 - 6 } Levels & Day 1 & Day 2 & Day 3 & Day 4 & Day 5 \\
\hline Level 1 & 48 & 45 & 45 & 47 & 49 \\
Level 2 & 34 & 33 & 35 & 35 & 39 \\
Level 3 & 30 & 39 & 33 & 34 & 34 \\
\hline
\end{tabular}

TABLE V

THE SCORE OF H4

\begin{tabular}{cccccc}
\hline Content & \multicolumn{5}{c}{ Training Date and Score } \\
\cline { 2 - 6 } Levels & Day 1 & Day 2 & Day 3 & Day 4 & Day 5 \\
\hline Level 1 & 45 & 47 & 49 & 45 & 48 \\
Level 2 & 39 & 42 & 40 & 44 & 45 \\
Level 3 & 32 & 30 & 35 & 33 & 36 \\
\hline
\end{tabular}

TABLE VI

THE SCORE OF H5

\begin{tabular}{cccccc}
\hline Content & \multicolumn{5}{c}{ Training Date and Score } \\
\cline { 2 - 6 } Levels & Day 1 & Day 2 & Day 3 & Day 4 & Day 5 \\
\hline Level 1 & 38 & 37 & 40 & 42 & 45 \\
Level 2 & 27 & 33 & 35 & 39 & 42 \\
Level 3 & 21 & 22 & 25 & 25 & 27 \\
\hline
\end{tabular}

TABLE VII

THE SCORE OF H6

\begin{tabular}{cccccc}
\hline Content & \multicolumn{5}{c}{ Training Date and Score } \\
\cline { 2 - 6 } Levels & Day 1 & Day 2 & Day 3 & Day 4 & Day 5 \\
\hline Level 1 & 33 & 38 & 37 & 38 & 38 \\
Level 2 & 28 & 26 & 27 & 29 & 29 \\
Level 3 & 14 & 17 & 16 & 20 & 21 \\
\hline
\end{tabular}

Table VIII is the score for each level of VR content divided into male and female. There were differences depending on the concentration of rehabilitation and visual acuity, but there were no differences by gender.

TABLE VIII

THE AVERAGE OF CONTENTS SCORE OF VR CONTENT

\begin{tabular}{cccc}
\hline \multirow{2}{*}{ Gender } & \multicolumn{3}{c}{ Average of Content Score of VR Content } \\
\cline { 2 - 4 } & Level 1 & Level 2 & Level 3 \\
\hline $\begin{array}{c}\text { Healthy } \\
\text { Male }(\mathrm{n}=3) \\
\begin{array}{c}\text { Healthy } \\
\text { Female }(\mathrm{n}=3)\end{array}\end{array}$ & 39.7 & 34.9 & 27.2 \\
\hline
\end{tabular}


Fig. 5 shows the results at content level 1. The distribution of scores was high for both female and male participants.

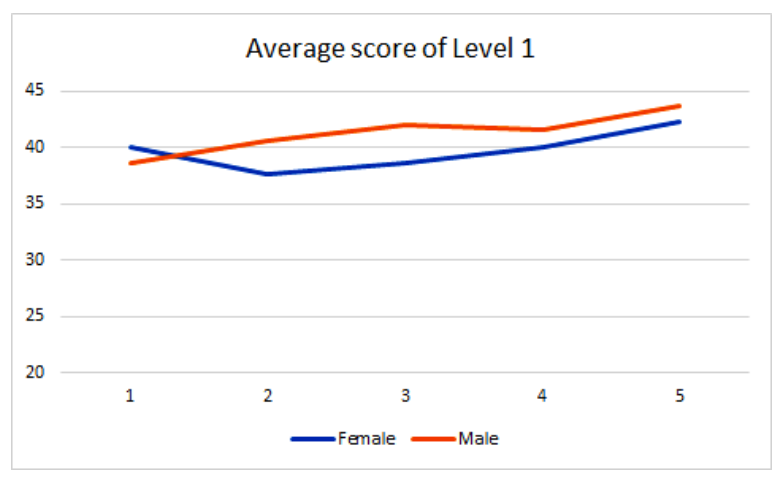

Fig. 5 The average score of Level 1

Compared to Level 2 in Fig. 6, Level 1 has a high score, and the score increase is not significant. On the other hand, Level 2 had a fairly low score in the early stages, which was not used to seeing it as a peripheral field, but gradually increased.

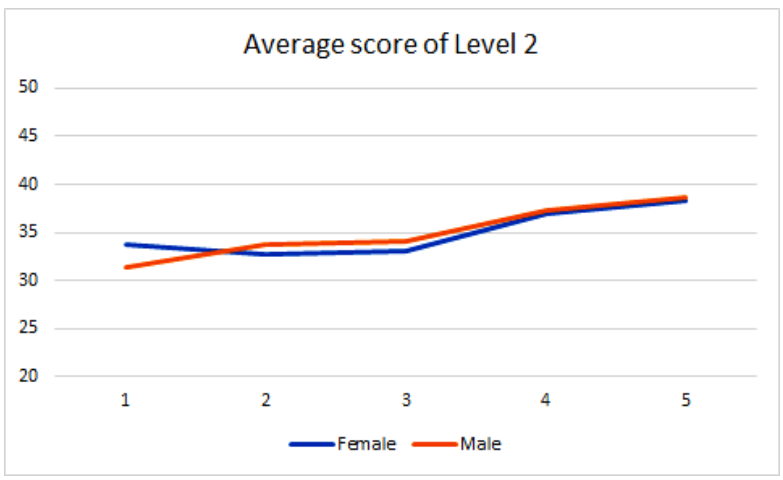

Fig. 6 The average score of Level 2

Level 3 in Fig. 7 has the lowest score overall. In the experiment on the second day, two of the participants in the experiment requested to change the previously selected area, and the change caused a large change in the score width.

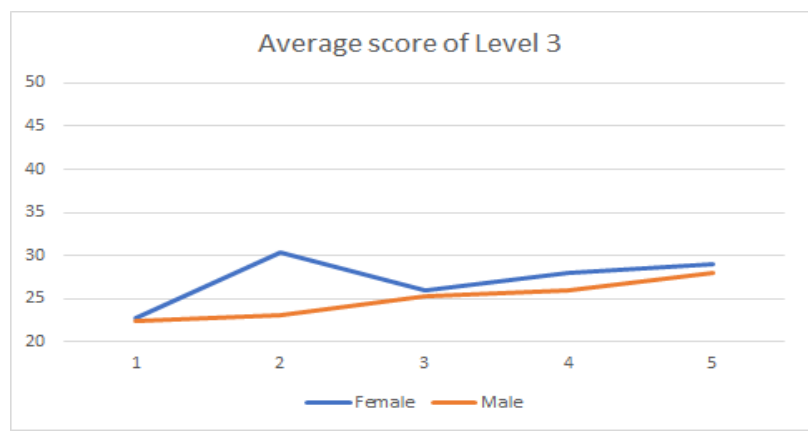

Fig. 7 The average score of Level 3

Overall, the participants' scores also increased in the level 3 experiment. In this way, it can be seen that if the adaptive training is performed after the location of the PRL is selected, the width of the score is improved according to the adaptability of the trainee.

\section{B. Result of PC Contents}

From Table IX to Table XIV is the result score of participants of PC content. The score was averaged after measuring the right and left eye scores separately.

TABLE IX

THE SCORE OF H7

\begin{tabular}{|c|c|c|c|c|c|}
\hline \multirow{2}{*}{$\begin{array}{l}\text { Content } \\
\text { Levels }\end{array}$} & \multicolumn{5}{|c|}{ Training Date and Score } \\
\hline & Day 1 & Day 2 & Day 3 & Day 4 & Day 5 \\
\hline Level 1 & 39 & 35 & 38 & 42 & 40 \\
\hline Level 2 & 23 & 22 & 21 & 25 & 27 \\
\hline Level 3 & 0 & 2 & 5 & 5 & 8 \\
\hline \multicolumn{6}{|c|}{$\begin{array}{c}\text { TABLE X } \\
\text { THE SCORE OF H8 }\end{array}$} \\
\hline \multirow{2}{*}{$\begin{array}{l}\text { Content } \\
\text { Levels }\end{array}$} & \multicolumn{5}{|c|}{ Training Date and Score } \\
\hline & Day 1 & Day 2 & Day 3 & Day 4 & Day 5 \\
\hline Level 1 & 35 & 35 & 32 & 34 & 33 \\
\hline Level 2 & 23 & 19 & 18 & 20 & 22 \\
\hline Level 3 & 9 & 9 & 8 & 10 & 12 \\
\hline \multicolumn{6}{|c|}{$\begin{array}{c}\text { TABLE XI } \\
\text { THE SCORE OF H9 }\end{array}$} \\
\hline \multirow{2}{*}{$\begin{array}{l}\text { Content } \\
\text { Levels }\end{array}$} & \multicolumn{5}{|c|}{ Training Date and Score } \\
\hline & Day 1 & Day 2 & Day 3 & Day 4 & Day 5 \\
\hline Level 1 & 42 & 45 & 47 & 47 & 50 \\
\hline Level 2 & 32 & 36 & 35 & 37 & 36 \\
\hline Level 3 & 12 & 10 & 15 & 16 & 15 \\
\hline \multicolumn{6}{|c|}{$\begin{array}{c}\text { TABLE XII } \\
\text { THE SCORE OF H10 }\end{array}$} \\
\hline \multirow{2}{*}{$\begin{array}{l}\text { Content } \\
\text { Levels }\end{array}$} & \multicolumn{5}{|c|}{ Training Date and Score } \\
\hline & Day 1 & Day 2 & Day 3 & Day 4 & Day 5 \\
\hline Level 1 & 41 & 40 & 42 & 45 & 48 \\
\hline Level 2 & 35 & 33 & 34 & 37 & 37 \\
\hline Level 3 & 15 & 12 & 15 & 15 & 16 \\
\hline \multicolumn{6}{|c|}{$\begin{array}{c}\text { TABLE XIII } \\
\text { THE SCORE OF H11 }\end{array}$} \\
\hline \multirow{2}{*}{$\begin{array}{l}\text { Content } \\
\text { Levels }\end{array}$} & \multicolumn{5}{|c|}{ Training Date and Score } \\
\hline & Day 1 & Day 2 & Day 3 & Day 4 & Day 5 \\
\hline Level 1 & 32 & 30 & 33 & 34 & 37 \\
\hline Level 2 & 30 & 33 & 37 & 37 & 36 \\
\hline Level 3 & 12 & 11 & 15 & 12 & 15 \\
\hline \multicolumn{6}{|c|}{$\begin{array}{c}\text { TABLE XIV } \\
\text { THE SCORE OF H12 }\end{array}$} \\
\hline \multirow{2}{*}{$\begin{array}{l}\text { Content } \\
\text { Levels }\end{array}$} & \multicolumn{5}{|c|}{ Training Date and Score } \\
\hline & Day 1 & Day 2 & Day 3 & Day 4 & Day 5 \\
\hline Level 1 & 35 & 40 & 45 & 45 & 49 \\
\hline Level 2 & 13 & 33 & 37 & 38 & 39 \\
\hline Level 3 & 0 & 3 & 2 & 3 & 4 \\
\hline
\end{tabular}

Table XV shows the average by dividing the PC content into stages and genders. When comparing with VR, you can see that Levels 1 and 2 scored high. However, when level 3 content was executed, all three participants complained of high eye fatigue.

TABLE XV

THE AVERAGE OF CONTENTS SCORE OF PC CONTENT

\begin{tabular}{cccc}
\hline \multirow{2}{*}{ Gender } & \multicolumn{3}{c}{ Average of Content Score of VR Content } \\
\cline { 2 - 4 } & Level 1 & Level 2 & Level 3 \\
\hline $\begin{array}{c}\text { Healthy Male } \\
(\mathrm{n}=3)\end{array}$ & 38.8 & 26.4 & 9.06 \\
$\begin{array}{c}\text { Healthy } \\
\text { Female }(\mathrm{n}=3)\end{array}$ & 39.7 & 33.9 & 10.0 \\
\hline
\end{tabular}




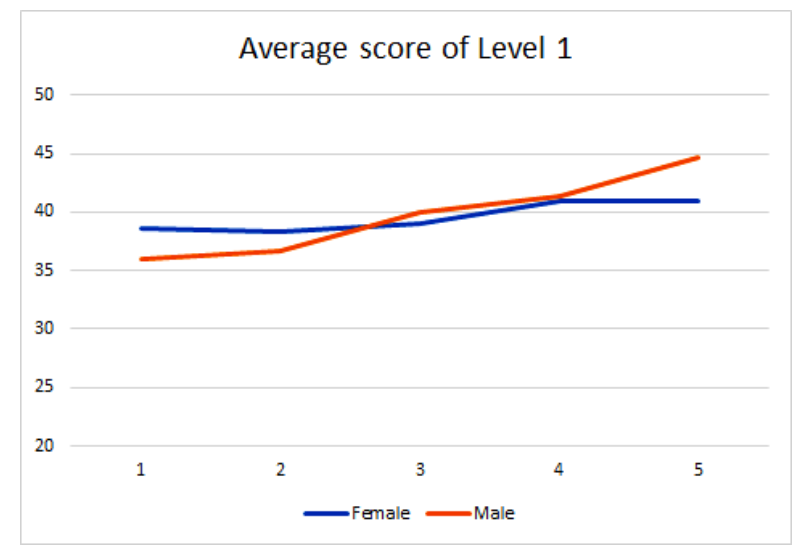

Fig. 8 The average score of Level 1

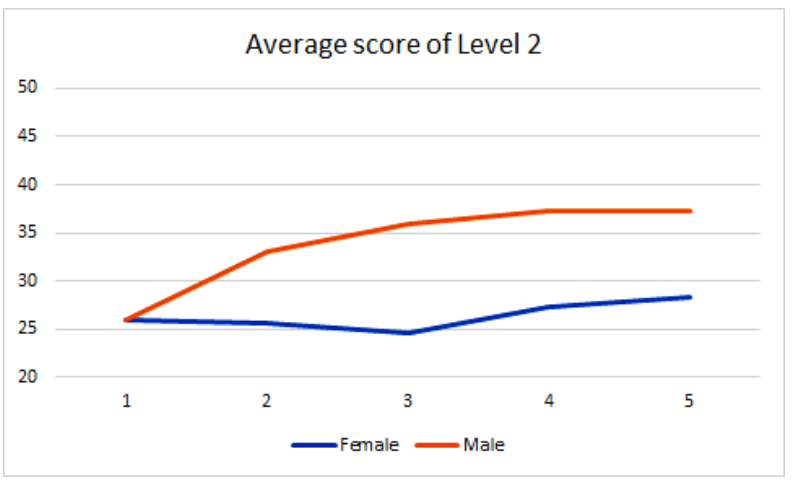

Fig. 9 The average score of Level 2

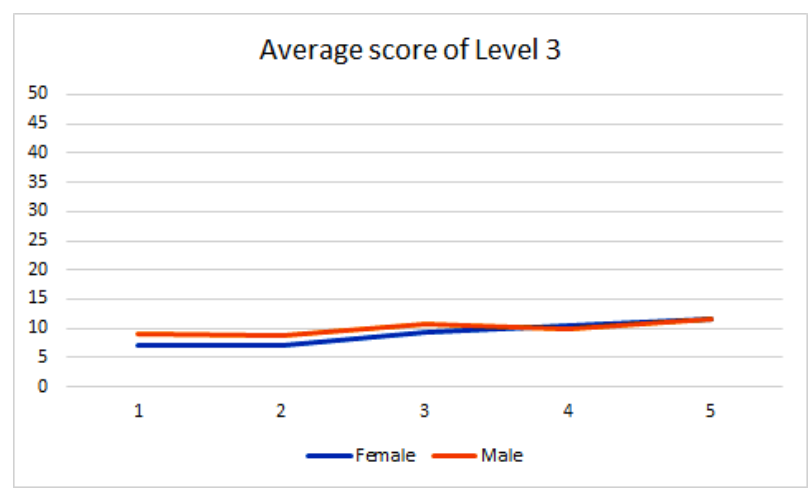

Fig. 10 The average score of Level 3

Fig. 8 is the result at content level 1. The distribution of scores was high for both female and male participants. Compared to Level 2 in Fig. 9, Level 1 scores high, and the increase in score is not significant. On the other hand, in Level 2 (Fig. 9), the male showed a large imagination in the 2nd to 4th experiment, but the female gradually increased the score. Level 3 of fig. 10 has the lowest score overall. Also, the score did not increase significantly over time. Also, in the case of Level 3, it was found that the concentration of the experiment participants was reduced considerably. The low score of level 3 predicted that the immersion of contents was also reduced because it was impossible to block the external environment completely, and the participants' fatigue was also high. In the case of pc content, the width of the score also increased, and all the participants showed an adaptation to RPL.

\section{CONCLUSION}

In this study, the contents that were conducted in the same way were implemented in PC and VR, respectively, and experiments were conducted. Because the PC version of rehabilitation content is a home-based vision therapy that can be rehabilitated at home, it has the advantage of being able to proceed regardless of time and place to maintain the effect of permanent rehabilitation. However, to maximize rehabilitation effectiveness, an assistant is required, and the assistant act's competency and role are important factors.

On the other hand, rehabilitation through VR can maximize the effectiveness of rehabilitation while minimizing the assistance of assistants while immersing in the rehabilitation process with the HMD completely blocked. When using an HMD with built-in EYE Tracking function such as FOVE, it is possible to easily collect the user's eye data, so more effective rehabilitation can be expected through data-based feedback.

In the experiment conducted through the two types of contents implemented, 12 non-disabled 6 men and 6 women participated as experiment participants. Before proceeding with the experiment through content, the variable characteristics of the PRL appearing in the process of determining the PRL and, in the case of not matching the purpose of the experiment, were excluded from the experimental result data, and the experiment was conducted.

This content was produced in two versions to test the hypothesis that fixation stability improves the target's attention and reading. As a result of the experiment, all participants showed an increase in the anchorage of the selected PRL on average, and it seemed to be adapted to seeing objects with PRL. Since the experiment was not conducted for a long time, and the experiment was conducted with a single content using only the target and size of the arrow rather than various contents, future research plans to diversify the content and difficulty further, and we want to apply this content directly to macular degeneration.

\section{ACKNOWLEDGMENT}

This research was financially supported by the "ICT Convergence Smart Rehabilitation Industrial Education Program" through the Ministry of Trade, Industry \& Energy (MOTIE). The authors are grateful to Korea Institute for Advancement of Technology (KIAT) and the MSIP (Ministry of Science, ICT and Future Planning), Korea, under the ITRC (Information Technology Research Center) support program (IITP-2020-2015-0-00403) supervised by the IITP (Institute for Information \& communications Technology Promotion).

\section{REFERENCES}

[1] Morales, M. U., Saker, S., Wilde, C., Rubinstein, M., Limoli, P., \& Amoaku, W. M. Biofeedback fixation training method for improving eccentric vision in patients with loss of foveal function secondary to different maculopathies. International Ophthalmology, 40(2), 305312, 2020.

[2] Jae-Myoung Seo, Study on the change of the peripheral visual function following eccentric viewing training, 2014.

[3] Hong, S. P. Effects of the Eccentric Viewing Training Software Based on Korean for Reading Speed: A Case Study. Journal of Digital Convergence, 16(7), 441-449, 2018. 
[4] Chung, S. T. Reading in the presence of macular disease: a mini-review. Ophthalmic and Physiological Optics, 2020.

[5] Gonçalves, Patrik, Jason Orlosky, and Tonja-Katrin Machulla. An Augmented Reality Assistant to Support Button Selection for Patients with Age-related Macular Degeneration. 2020 IEEE Conference on Virtual Reality and 3D User Interfaces Abstracts and Workshops (VRW). IEEE, 2020.

[6] Burge, W. K., Griffis, J. C., Nenert, R., Elkhetali, A., DeCarlo, D. K., Lawrence, W., ... \& Visscher, K. M. Cortical thickness in human VI associated with central vision loss. Scientific reports, 6(1), 1-10, 2016

[7] Ramanoel, S., Chokron, S., Hera, R., Kauffmann, L., Chiquet, C., Krainik, A., \& Peyrin, C.. Age-related macular degeneration changes the processing of visual scenes in the brain. Visual neuroscience, 35, 2018.

[8] García-Layana, A., Cabrera-Lopez, F., García-Arumí, J., AriasBarquet, L., \& Ruiz-Moreno, J. M.. Early and intermediate agerelated macular degeneration: update and clinical review. Clinical interventions in aging, 12, 1579, 2017

[9] O'connell, C., Mahboobin, A., Drexler, S., Redfern, M. S., Perera, S. Nau, A. C., \& Cham, R.. Effects of acute peripheral/central visual field loss on standing balance. Experimental brain research, 235(11), 3261-3270, 2017.

[10] Estudillo, J. A. R., Higuera, M. I. L., Juárez, S. R., Vera, M. D. L. O., Santana, Y. P., \& Suazo, B. C. Visual rehabilitation via microperimetry in patients with geographic atrophy: a pilot study. International journal of retina and vitreous, 3(1), 21, 2017.

[11] Macnaughton, Jane, Keziah Latham, and Marta Vianya-Estopa Rehabilitation needs and activity limitations of adults with a visual impairment entering a low vision rehabilitation service in England. Ophthalmic and physiological optics 39.2, 113-126, 2019.

[12] Selivanova, A., Fenwick, E., Man, R., Seiple, W., \& Jackson, M. L. Outcomes After Comprehensive Vision Rehabilitation Using Visionrelated Quality of Life Questionnaires: Impact of Vision Impairment and National Eye Institute Visual Functioning Ouestionnaire. Optometry and Vision Science, 96(2), 87-94, 2019.

[13] Bittner, A. K., Yoshinaga, P., Bowers, A., Shepherd, J. D., Succar, T., \& Ross, N. C. Feasibility of telerehabilitation for low vision: satisfaction ratings by providers and patients. Optometry and Vision Science, 95(9), 865-872, 2018.

[14] Silvestri, Valeria. Eye-Fitness: A Home-Based Rehabilitation Program for People with Central Vision Loss. EC Ophthalmol 10 , 649-659, 2019.

[15] Flaxman, S. R., Bourne, R. R., Resnikoff, S., Ackland, P., Braithwaite, T., Cicinelli, M. V., ... \& Leasher, J. Global causes of blindness and distance vision impairment 1990-2020: a systematic review and meta-analysis. The Lancet Global Health, 5(12), e1221e1234, 2017.

[16] Kim, S., Park, S. J., Byun, S. J., Park, K. H., \& Suh, H. S. Incremental economic burden associated with exudative age-related macular degeneration: a population-based study. BMC health services research, 19(1), 828, 2019.

[17] Taylor, D. J., Hobby, A. E., Binns, A. M., \& Crabb, D. P. How does age-related macular degeneration affect real-world visual ability and quality of life? A systematic review. BMJ open, 6(12), e011504, 2016.

[18] Roh, M., Selivanova, A., Shin, H. J., Miller, J. W., \& Jackson, M. L. Visual acuity and contrast sensitivity are two important factors affecting vision-related quality of life in advanced age-related macular degeneration. PloS one, 13(5), 2018.

[19] Wallis, T. S., Taylor, C. P., Wallis, J., Jackson, M. L., \& Bex, P. J. Characterization of field loss based on microperimetry is predictive of face recognition difficulties. Investigative ophthalmology \& visual science, 55(1), 142-153, 2014

[20] Taylor, D. J., Jones, L., Binns, A. M., \& Crabb, D. P. 'You've got dry macular degeneration, end of story': a qualitative study into the experience of living with non-neovascular age-related macular degeneration. Eye, 34(3), 461-473, 2020.
[21] Rose, Dylan, and Peter Bex. Peripheral oculomotor training in individuals with healthy visual systems: effects of training and training transfer. Vision Research 133, 95-99, 2017.

[22] Treleaven AJ, Yu D. Training peripheral vision to read: reducing crowding through an adaptive training method. Vision research. 1;171:84-94, Jun 2020.

[23] Markowitz, M., Daibert-Nido, M., \& Markowitz, S. N. Rehabilitation of reading skills in patients with age-related macular degeneration. Canadian Journal of Ophthalmology, 53(1), 3-8, 2018.

[24] Crossland, Michael D., Stephen A. Engel, and Gordon E. Legge. The preferred retinal locus in macular disease: toward a consensus definition. Retina 31.10, 2109-2114, 2011.

[25] Altınbay, Deniz, and Şefay Aysun İdil. Current Approaches to Low Vision (Re) Habilitation. Turkish journal of ophthalmology 49.3, 154, 2019.

[26] Freeman, D., Reeve, S., Robinson, A., Ehlers, A., Clark, D., Spanlang, B., \& Slater, M. Virtual reality in the assessment, understanding and treatment of mental health disorders. Psychological medicine, 47(14), 2393-2400, 2017.

[27] Riva, Giuseppe, Brenda K. Wiederhold, and Fabrizia Mantovani. Neuroscience of virtual reality: From virtual exposure to embodied medicine. Cyberpsychology, Behavior, and Social Networking 22.1, 82-96, 2019.

[28] Powell, Wendy, Vaughan Powell, and Marc Cook. The Accessibility of Commercial Off-The-Shelf Virtual Reality for Low Vision Users: A Macular Degeneration Case Study. Cyberpsychology, Behavior, and Social Networking 23.3, 185-191, 2020.

[29] De Lange C. Vision restored with virtual reality. New Scientist, 239:4, 2018

[30] Maggio, M. G., De Luca, R., Molonia, F., Porcari, B., Destro, M. Casella, C., ... \& Calabro, R. S. Cognitive rehabilitation in patients with traumatic brain injury: A narrative review on the emerging use of virtual reality. Journal of Clinical Neuroscience, 61, 1-4, 2019.

[31] Howard, M. C. A meta-analysis and systematic literature review of virtual reality rehabilitation programs. Computers in Human Behavior, 70, 317-327, 2017.

[32] Ehrlich, J. R., Ojeda, L. V., Wicker, D., Day, S., Howson, A., Lakshminarayanan, V., \& Moroi, S. E. Head-mounted display technology for low-vision rehabilitation and vision enhancement American journal of ophthalmology, 176, 26-32, 2017.

[33] Prahalad, Krishnamachari S., and Daniel R. Coates. Asymmetries of reading eye movements in simulated central vision loss. Vision Research 171, 1-10, 2020.

[34] Maniglia, M., Pavan, A., Sato, G., Contemori, G., Montemurro, S., Battaglini, L., \& Casco, C. Perceptual learning leads to long lasting visual improvement in patients with central vision loss. Restorative neurology and neuroscience, 34(5), 697-720, 2016.

[35] Daibert-Nido, M., Patino, B., Markowitz, M., \& Markowitz, S. N. Rehabilitation with biofeedback training in age-related macular degeneration for improving distance vision. Canadian Journal of Ophthalmology, 54(3), 328-334, 2019.

[36] Jeong, J. H., \& Moon, N. J. A study of eccentric viewing training for low vision rehabilitation. Korean Journal of Ophthalmology, 25(6), 409-416, 2011.

[37] Calabrèse, Aurélie, Tingting Liu, and Gordon E. Legge. Does vertical reading help people with macular degeneration: an exploratory study. PloS one 12.1, 2017.

[38] Yu, D., Legge, G. E., Park, H., Gage, E., \& Chung, S. T. Development of a training protocol to improve reading performance in peripheral vision. Vision Research Vol. 50, No.1, pp. 36-45, 2010.

[39] Chow-Wing-Bom, Hugo, Tessa M. Dekker, and Pete R. Jones. The worse eye revisited: evaluating the impact of asymmetric peripheral vision loss on everyday function. Vision Research 169, 2020, 49-57.

[40] Chen, N., Shin, K., Millin, R., Song, Y., Kwon, M., \& Tjan, B. S. Cortical reorganization of peripheral vision induced by simulated central vision loss. Journal of Neuroscience, 39(18), 3529-3536, 2019 